\title{
A current perspective of autophagosome biogenesis
}

\author{
Shusaku T Shibutani, ${ }^{1,2}$, Tamotsu Yoshimori, ${ }^{1,2}$ \\ ${ }^{1}$ Laboratory of Intracellular Membrane Dynamics, Graduate School of Frontier Biosciences, Osaka University, Osaka 565-0871, \\ Japan; ${ }^{2}$ Department of Genetics, Graduate School of Medicine, Osaka University, Osaka 565-0871, Japan
}

Autophagy is a bulk degradation system induced by cellular stresses such as nutrient starvation. Its function relies on the formation of double-membrane vesicles called autophagosomes. Unlike other organelles that appear to stably exist in the cell, autophagosomes are formed on demand, and once their formation is initiated, it proceeds surprisingly rapidly. How and where this dynamic autophagosome formation takes place has been a long-standing question, but the discovery of Atg proteins in the 1990's significantly accelerated our understanding of autophagosome biogenesis. In this review, we will briefly introduce each Atg functional unit in relation to autophagosome biogenesis, and then discuss the origin of the autophagosomal membrane with an introduction to selected recent studies addressing this problem.

Keywords: autophagy; autophagosome; Atg

Cell Research (2014) 24:58-68. doi:10.1038/cr.2013.159; published online 3 December 2013

\section{Introduction}

Macroautophagy, usually referred to simply as autophagy, is an intracellular degradation system conserved among eukaryotes from yeast to human. A prominent feature of autophagy is the dynamic membrane reorganization that results in the formation of double-membrane autophagosomes. During autophagy, a cup-shaped double-membrane sac called the isolation membrane (also called the phagophore) emerges in the cytoplasm. The isolation membrane expands in size, and its open end eventually closes to form a double-membrane vesicle called the autophagosome (Figure 1). After the completion of autophagosome formation, the outer membrane of the autophagosome fuses with a lysosome (in mammals) or the vacuole (a lysosome-like organelle in yeast), and the contents inside the autophagosome and its inner membrane are degraded by lysosomal hydrolases. These processes proceed rapidly. In yeast, it is estimated that autophagosome formation takes 4-5 min, and one autophagy cycle from autophagosome initiation to its degradation takes 7-9 min [1]. In mammals, it is estimated

Correspondence: Tamotsu Yoshimori

Tel: +81-6-6879-3580; Fax: +81-6-6879-3589

E-mail: tamyoshi@fbs.osaka-u.ac.jp that autophagosome formation takes 5-10 min $[2,3]$.

Autophagosome formation is highly inducible. Deprivation of nutrients, especially amino acid, is a wellknown inducer of autophagy. In nutrient-rich conditions, the protein kinase complex TORC1/mTORC1 suppresses autophagosome formation. Inversely, nutrient starvation leads to autophagy induction through TORC1/mTORC1 suppression. TORC1/mTORC1 inhibitors such as rapamycin can induce autophagy even under nutrient-rich conditions. Autophagy induction leads to the degradation of cytosolic macromolecules, providing energy and building blocks for biosynthesis [4].

The diameter of the autophagosome is typically 0.3-0.9 $\mu \mathrm{m}$ in yeast [5] and 0.5-1.5 $\mu \mathrm{m}$ in mammals [6]. Since a relatively large amount of cytosolic components can

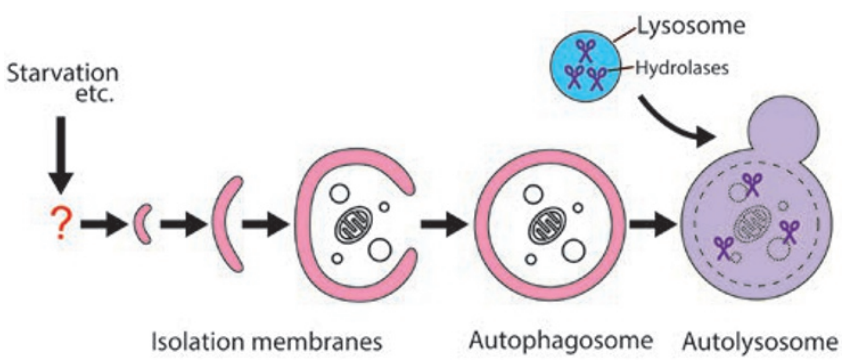

Figure 1 Schematic of autophagosome formation. 
be trapped in an autophagosome, autophagy can handle degradation of not only cytosolic macromolecules, but also much larger structures such as cell organelles. This is in marked contrast to the ubiquitin-proteasome system, which degrades individual ubiquitinated proteins. Recent studies suggest that autophagy maintains intracellular homeostasis by eliminating unwanted molecules/structures such as protein aggregates, damaged mitochondria, excessive peroxisomes, ribosomes, and invading pathogens [7]. Although autophagy was initially thought of as a non-specific degradation system, it exhibits selectivity in these cases. This type of autophagy, called selective autophagy, will be discussed in the last part of this review.

Consistent with the idea that autophagy is generally beneficial for the cell, systemic autophagy-deficient mice die within a day after birth [8-12], and oocyte-specific autophagy deficiency results in lethality during early embryogenesis [13]. Moreover, it is becoming apparent that defects in autophagy are associated with many dysfunctions, such as tumor development, neurodegenerative diseases, cardiac hypertrophy, diabetes, and pathogen infection, as well as abnormal differentiation in many cell lineages [14]. However, despite the apparent biological and clinical importance of autophagy, we are just beginning to understand the molecular mechanisms of autophagosome formation.

\section{Atg proteins}

Observations of autophagy-related structures by electron microscopy have been reported since the 1950's, and early studies showed that autophagy is induced in rat livers by glucagon or by amino acid depletion, and is suppressed by insulin [15]. Despite this, the molecular mechanisms of autophagy remained largely unknown. The discovery of autophagy-related $(A T G)$ genes in the 1990 's by yeast genetics provided powerful genetic and molecular tools to investigate autophagy [16]. To date, over $35 A T G$ genes have been identified in yeast. A total of 15 core $A T G$ genes required for both starvationinduced and selective autophagy (ATG1-10, 12-14, 16, 18) are highly conserved in mammals [17].

In yeast, autophagosomes are formed at a single site called the pre-autophagosomal structure (PAS; also called phagophore assembly site) [18]. Under a fluorescence microscope, the PAS is detected as a single, dot-like accumulation of Atg proteins next to the vacuole. Epistasis analysis using atg mutants went on to reveal the hierarchical relationships among these Atg proteins [19]. In mammals, autophagosome formation sites (the counterpart of the yeast PAS) have also been characterized as dot-like accumulations of Atg proteins at multiple locations in a single cell, and have a hierarchy similar to yeast Atg proteins [20].

A natural question to ask, then, is how the Atg proteins function to generate autophagosomes. The core Atg proteins can be functionally categorized into several units: the Atg1/ULK complex, the class III phosphatidylinositol 3-kinase (PI3K) complex, the Atg2-Atg18/ WIPI complex, the Atg12 conjugation system, the Atg8/ LC 3 conjugation system, and Atg9 vesicles. Understanding Atg proteins will provide insights into a question that existed long before the discovery of Atg proteins: what is the origin of the autophagosome? Or, more specifically, where do the lipid molecules for autophagosomal membrane biogenesis come from? In the next sections, each Atg functional unit will be briefly discussed in relation to autophagosome biogenesis, followed by a discussion of the origin of the autophagosome.

\section{The Atg1/ULK complex}

The Atg1/ULK complex is placed at the most upstream position in the hierarchy of Atg protein recruitment [19, 21]. In canonical autophagy in yeast, a 2:2:2 complex formed by Atg17-Atg31-Atg29 functions as the scaffolding subunits for the Atg1 complex [22]. Upon starvation, decreased TORC1 activity results in dephosphorylation of Atg13, leading to the incorporation of Atg1-Atg13 into the Atg17-Atg29-Atg31 complex [23, 24]. In mammals, the orthologues of Atg1 (ULK1 and 2; of the two, ULK1 is thought to play the major role in autophagy $[25,26]$ ) and $A \operatorname{tg} 13$ exist, but those of $A \operatorname{tg} 17, \operatorname{Atg} 29$, and Atg31 have not been identified. Instead, FIP200 and Atg101 are thought of as the functional counterparts of yeast Atg17-Atg29-Atg31. Yeast Atg1 and mammalian ULKs are Ser/Thr kinases. Although the kinase activity of Atg1 is required for canonical autophagy [24], it has been shown that downstream Atg proteins still accumulate in yeast cells with a kinase-deficient mutation of Atg1 $[27,28]$. On the other hand, in mammals, expression of kinase-deficient mutants of ULK1 and ULK2 leads to a dominant negative effect and suppresses accumulation of downstream Atg proteins $[21,29]$. Taken together, the Atg1/ULK complex functions as the scaffold for the recruitment of downstream Atg proteins, and in yeast, the scaffolding function does not require the kinase activity of Atg1.

\section{The class III PI3K complex and the Atg2-Atg18/ WIPI complex}

Autophagosomal membranes contain a specific lipid molecule, phosphatidylinositol 3-phosphate (PI3P) [30]. 
Consistently, autophagosome formation requires the class III PI3K complex, which produces PI3P by phosphorylating phosphatidylinositol (PI) at the 3-position hydroxyl group of the inositol ring. In yeast, the autophagy-specific PI3K complex is composed of four proteins: Vps34 (catalytic unit), Vps15, Atg6/Vps30, and Atg14 [31]. In mammals, Beclin $1[32,33]$ and Atg14L/Barkor [34$37]$ are identified as the orthologues of $\operatorname{Atg} 6 / \mathrm{Vps} 30$ and Atg14, respectively. It is thought that the production of PI3P is initiated by the dot-like accumulation of the Atg14/Atg14L-containing PI3K complex at the PAS (in yeast) or on the endoplasmic reticulum (ER) (in mammals; discussed below). The accumulation of PI3P generates a platform to recruit PI3P-binding proteins. The PI3P-binding protein Atg18/WIPI and its binding partner Atg2 are recruited to such a platform. Although the exact function of Atg2-Atg18/WIPI in autophagosome biogenesis is not clear, it has been shown that the Atg2-Atg18 complex is required for normal distribution of Atg9 vesicles [38-40].

\section{The Atg12 conjugation system}

Two ubiquitin-like conjugation systems exist in the core machinery of autophagy. In the Atg12 conjugation system, the C-terminal glycine of Atg12 is covalently conjugated to the lysine residue located in the middle of Atg5 [41]. In this system, Atg7 and Atg10 catalyze this conjugation by functioning as E1-like and E2-like enzymes, respectively, but no E3-like enzymes for this system appear to exist. The Atg12-Atg5 complex binds to Atg16 (Atg16L1 and 2 in mammals; Atg16L1 is thought to be the major form in autophagy [42]) through the direct association of Atg16(L1) and Atg5. Atg12-Atg5Atg16(L1) forms a 2:2:2 complex through the homodimerization domain of Atg16(L1) [43, 44]. The Atg12Atg5-Atg16(L1) complex localizes to the isolation membrane and its precursor, and dissociates from the membrane upon the completion of autophagosome formation $[2,18]$. Therefore, $\operatorname{Atg} 12, \operatorname{Atg} 5$, and $\operatorname{Atg} 16(\mathrm{~L} 1)$ are frequently used as markers for the early steps of autophagosome formation. The localization of the Atg12-Atg5Atg16(L1) complex appears to be directed by Atg16(L1) $[19,45]$, and it has been shown that mammalian Atg16L1 directly binds to the ULK1 complex subunit FIP200 [4648]. In addition, the membrane-binding ability of yeast Atg5, which can be inhibited by $\operatorname{Atg} 12$ and activated by Atg16, has also been reported [49]. The Atg12-Atg5Atg16 complex functions as the E3-like enzyme for the other ubiquitin-like conjugation system: the Atg8/LC3 conjugation system [50].

\section{The Atg8/LC3 conjugation system}

Yeast $\operatorname{Atg} 8$ and the mammalian Atg 8 orthologues (LC3A/B/C, GABARAP, and GABARAPL1/2/3; hereafter represented by LC3) are not conjugated to proteins, but to the lipid molecule phosphatidylethanolamine (PE). Atg8/LC3 is translated as a precursor. Shortly after translation, the C-terminal peptide of the precursor is truncated by Atg4, and the C-terminal glycine of Atg8/LC3 is exposed [51]. The C-terminal glycine is conjugated to the amino group of PE, and Atg7 and Atg3 act as the E1like and E2-like enzymes for this conjugation [52]. The Atg12-Atg5-Atg16 complex acts as the E3-like enzyme [50]. Since the Atg12-Atg5-Atg16(L1) complex accumulates on the isolation membrane and its precursor, $\operatorname{Atg} 8 /$ LC3-PE conjugation takes place on these membranes. PE-conjugated Atg8/LC3 stably associates with autophagy-related structures, including isolation membranes and completed autophagosomes, and is therefore widely used as a marker for microscopic analysis of autophagy $[53,54]$. In addition, PE-conjugated Atg8/LC3 displays increased mobility in SDS-PAGE gels compared to its unconjugated form, and can be specifically detected by western blotting. It has been shown that the amount of PE-conjugated LC3 (also called LC3-II, as opposed to unconjugated LC3-I) correlates well with the number of autophagosomes/autolysosomes [54], and therefore the amount of LC3-II is widely used for quantification of autophagic activity. Even though it is a widely used marker, the exact function of Atg8/LC3 is unclear. In vitro analysis using PE-containing liposomes and purified Atg8 has shown that Atg8-PE can facilitate hemifusion of liposomes [55]. In mammals, suppression of PE-conjugation of the Atg8 orthologues by overexpression of a dominant negative form of $\mathrm{Atg} 4\left(\mathrm{Atg} 4 \mathrm{~B}^{\mathrm{C} 74 \mathrm{~A}}\right)$ [3] or by $\mathrm{Atg} 3 \mathrm{knock}-$ out [11] resulted in accumulation of unclosed isolation membranes, suggesting that Atg8/LC3 may function during the closure of the isolation membrane.

\section{Atg9 vesicles}

Atg9 is the only transmembrane protein among the core Atg proteins. Although Atg9 is required for the accumulation of most of the downstream Atg proteins [19, 56], its function is still enigmatic. In yeast, Atg9 has been shown to localize on small vesicles of 30-60 nm diameter (Atg9 vesicles) [57], which localize to the PAS and in the cytoplasm. The PAS localization of Atg9 requires postGolgi vesicle-trafficking machinery [58-62]. Yeast Atg9 was also found on isolation membranes and autophagosomes [57]. In mammals, Atg9 partially localizes to the trans-Golgi network and endosomes, and starvation 
induces the exit of Atg9 vesicles from the trans-Golgi network and transient colocalization of Atg9 with the autophagosome marker LC3 when visualized by fluorescence microscopy [26]. Mammalian Atg9 was detected on small vesicles and tubular structures similarly to yeast Atg9 [40]. Since Atg9 localizes to membrane vesicles, it was thought that these vesicles may supply membrane for autophagosome biogenesis. This possibility will be discussed later in this review.

\section{Searching for the origin of the autophagosomal mem- brane}

The discovery of Atg proteins provided us with powerful tools to investigate the mechanism of autophagosome biogenesis. Recent studies significantly advanced our knowledge about the origin of the autophagosomal membrane, especially in mammals. As discussed below, the ER is a strong candidate for the membrane source. In addition, mitochondria, the ER-Golgi intermediate compartment (ERGIC), the Golgi apparatus (often in relationship with Atg9 vesicles), recycling endosomes, and the plasma membrane are all proposed sources of the autophagosomal membrane.

\section{Autophagosome formation on the ER}

Recent studies using mammalian cells revealed a strong relationship between autophagosome formation sites and the ER. Axe et al. [63] investigated a protein called double FYVE domain containing protein 1 (DFCP1), which binds to PI3P through its FYVE domains. Unlike the typical endosomal localization of other PI3P-binding proteins, DFCP1 localizes to the ER and Golgi. The au- thors also found that starvation induced multiple dot-like structures of DFCP1 on the ER. Further analysis indicated that LC3-positive isolation membranes emerge from the centers of DFCP1-positive, $\sim 1 \mu \mathrm{m}$ diameter ring-like structures. These were named omegasomes, as DFCP1positive rings often looked like $\Omega$-shaped structures on the ER. The connection between isolation membranes and the ER was further confirmed by two independent studies using 3D tomographic imaging of isolation membranes $[64,65]$. These studies have shown that the cupshaped isolation membrane was tightly sandwiched by two sheets of ER. Moreover, the isolation membrane was physically connected to the ER through a narrow membrane tube. Therefore, isolation membrane formation and elongation may be guided by the adjacent ER sheets. This "ER cradle model" was further supported by the finding that $70 \%$ of autophagosomes contained portions of the ER [64]. Importantly, the isolation membrane/ER complex was labeled by DFCP1 [64], suggesting that the omegasome and the ER cradle may be identical structures (Figure 2).

The PI3K inhibitors, 3-methyladenine and wortmannin, inhibit the formation of omegasomes [63], indicating that the omegasome, where autophagosome formation takes place, is the site of local PI3P production on the ER. Consistently, Atg14L, a subunit of the autophagyspecific PI3K, was shown to localize to the ER by fluorescence and immuno-electron microscopy [35]. Under resting conditions, Atg14L evenly localized to the ER, and during starvation, $\operatorname{Atg} 14 \mathrm{~L}$ formed punctate structures on the ER. Further investigation revealed that the N-terminal region of Atg14L contains a conserved cysteine repeat that is required for its ER localization. Since the mutation of the cysteine repeat abrogated the

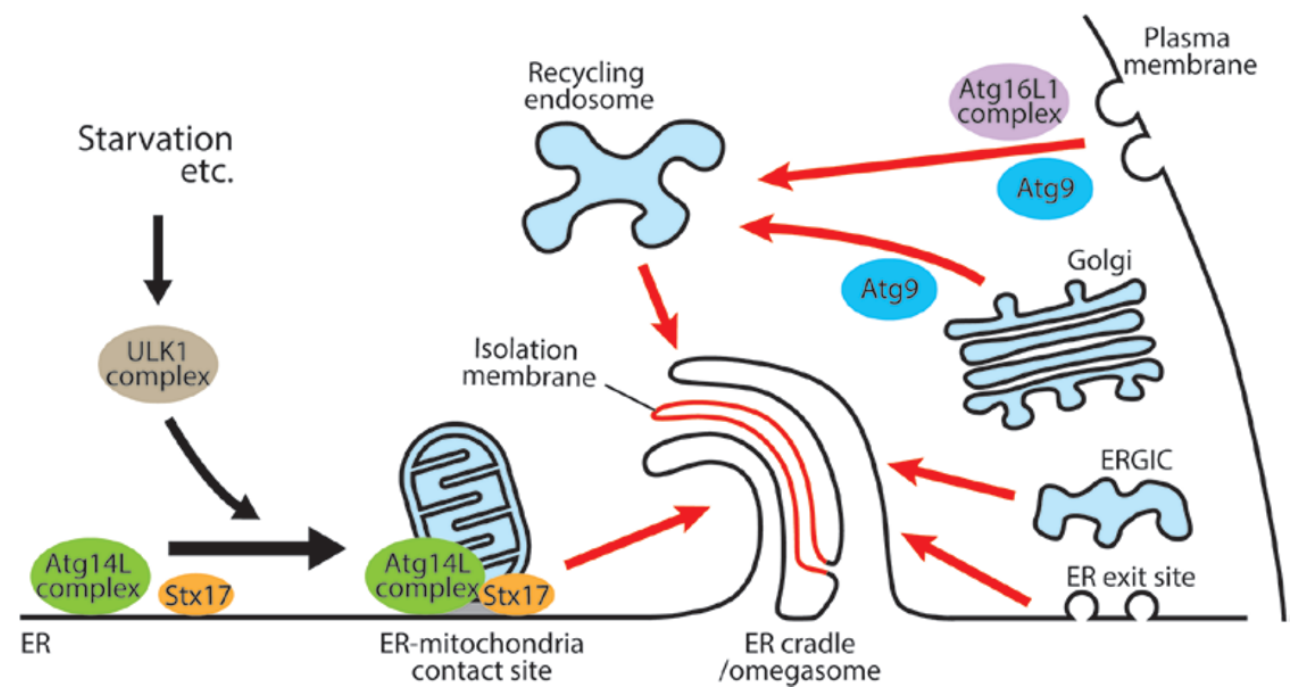

Figure 2 Proposed membrane sources of autophagosomal membrane. Upon starvation, Syntaxin-17 (Stx17) facilitates Atg14L accumulation at ERmitochondria contact sites, inducing the formation of the DFCP1-positive ER cradle/ omegasome on the ER. In this model, other membrane sources support the elongation of the forming isolation membrane at the ER cradle/ omegasome. 
autophagy-inducing ability of $A \operatorname{tg} 14 \mathrm{~L}$, and the addition of the ER localization sequence from DFCP1 rescued the deficiency, it was concluded that the ER localization of Atg14L is crucial for autophagosome biogenesis [66]. In addition, the C-terminus of mammalian $\operatorname{Atg} 14 \mathrm{~L}$, which is also required for its ER localization [66], has been shown to preferentially bind to liposomes containing PI3P or $\mathrm{PI}(4,5) \mathrm{P} 2$ [67].

Consistent with Atg14L dot formation on the ER, it was found that ULK1 and Atg5 also form dot-like accumulations on the ER, and that these dots are also associated with the ER when visualized by live cell imaging [20, 68]. Recently, Koyama-Honda et al. [69] have shown that ULK1 and Atg5 are simultaneously recruited and accumulate to form dots upon starvation, possibly through the direct interaction between FIP200 and Atg16L1 [4648]. Shortly after ULK1/Atg5 dot formation, Atg14L accumulates to form a dot overlapping the ULK1/Atg5 dot, possibly through an association of ULK1 and Atg14L mediated by the Exo84 exocyst complex [70]. Although the ULK1 complex is placed upstream of the PI3K complex [20, 66], PI3K activity appears to stabilize ULK1/ Atg5 dot formation $[69,71]$. In addition, it was shown that most ULK1 dots are formed on pre-existing VMP1 dots [69]. VMP1 has been previously shown to form dot-like accumulations on the ER, in addition to the ER network and the Golgi, regardless of nutrient conditions [20]. VMP1 was originally identified in C. elegans using a screen designed to identify eukaryotic autophagy genes [72]. Since VMP1 dots can be seen without autophagy induction and therefore precedes the accumulation of other Atg proteins, VMP1 may be marking autophagosome formation sites. However, VMP1 knockdown/ knockout does not prevent the early steps of autophagy, and instead leads to aberrant accumulation of Atg proteins, suggesting that VMP1 functions in a late step even though its dot formation precedes recruitment of other Atg proteins [20].

\section{ER-mitochondria contact sites}

In contrast, a study by Hailey et al. [73] suggested that the outer mitochondrial membrane contributes to autophagosome biogenesis in starved mammalian cells. The authors demonstrated that a protein in the outer mitochondrial membrane (YFP-Mito ${ }^{\mathrm{cb} 5}$ TM: YFP fused to the targeting sequence of a mitochondria-specific isoform of cytochrome b5) colocalized with LC3-positive membranes (isolation membranes and autophagosomes) after starvation. However, markers for the mitochondrial matrix or inner mitochondrial membrane did not colocalize with LC3-positive membranes even after starva- tion. Interestingly, even outer mitochondrial membrane proteins did not colocalize with LC3-positive structures if the protein spanned the outer and inner leaflets (YFPMito ${ }^{\mathrm{cb5}} \mathrm{TM}$ is inserted only to the outer leaflet of the outer mitochondria membrane). The authors reasoned that the sharp membrane curvature at the connection site between the mitochondrion and the isolation membrane prevents the transfer of transmembrane proteins if they penetrate both the outer and inner leaflets.

Therefore, two models of the origin of the autophagosome existed: the ER and mitochondria. These two separate models, however, may be reconciled by the recent work by Hamasaki et al. [68], which focuses on the contact sites between the ER and mitochondria (Figure 2). ER-mitochondria contact sites were previously known to exist and play important roles in mitochondria fission, calcium signaling, lipid transfer and so on [74]. It is possible that YFP-Mito ${ }^{\mathrm{cb5} 5} \mathrm{TM}$ entered the nascent isolation membrane after passing through the ER-mitochondria contact site. Indeed, in mitofusin 2-knockout cells, in which the ER-mitochondria contact sites are disrupted, autophagosome formation was significantly suppressed [73]. The mitochondria-associated ER membrane (MAM), which includes ER-mitochondria contact sites, can be recovered by subcellular fractionation. Upon starvation, components of the autophagy-specific class III PI3K (Atg14L, Beclin1, Vps34, and Vps15) all accumulated in the MAM fraction. Atg5, which is downstream of $\operatorname{Atg} 14 \mathrm{~L}$, almost always formed dots on the ER and remained associated with the ER over time. These Atg5 dots were also associated with mitochondria, but the Atg5-mitochondria association fluctuates dynamically, explaining why only about half of the Atg 5 dots were found adjacent to mitochondria in fixed cells [68]. Consistent with the results of Hailey et al. obtained from mitofusin 2 knockout cells, disrupting the ER-mitochondria contact sites by knockdown of PACS-2 or mitofusin-2 inhibited Atg14L and LC3 dot formation, as well as LC3 lipidation [68]. Interestingly, syntaxin 17 (Stx17), an ERresident Qa-SNARE protein identified in a screen for SNARE proteins involved in autophagy against group A Streptococcus, is responsible for starvation-induced relocalization of Atg14L to the MAM fraction. Stx17 relocalized to the MAM fraction after starvation like Atg14L, and Stx17 co-immunoprecipitated with Atg14L, Vps34, Beclin 1, and Vps15 under starvation conditions. Stx17 knockdown did not prevent Atg14L dot formation, but instead resulted in Atg14L dot formation outside of ERmitochondria contact sites. In Stx17-knockdown cells, Atg5-positive autophagic structures accumulated, but no autolysosomes were observed [68]. Itakura et al. [75] independently identified Stx 17 as an autophagosomal 
SNARE that is required for starvation-induced autophagy. These authors showed that Stx17 has two tandem transmembrane domains that are sufficient for its localization to autophagosomes, likely through the formation of a hairpin-like conformation that can be inserted into the outer membrane of the autophagosome. They observed that Stx17 is relocated to the outer membrane of autophagosomes after completion of isolation membrane closure. Stx17, together with VAMP8 and SNAP29, facilitates fusion between autophagosomes and lysosomes [75]. Stx17 thus, may have two separate functions in autophagosome formation depending on the timing and location: ER-resident Stx17 may regulate the recruitment of Atg14L dots to ER-mitochondria contact sites, and Stx 17 inserted in the outer membrane of autophagosomes after completion of autophagosome formation may facilitate autophagosome-lysosome fusion.

\section{ER exit sites}

Recently, Suzuki et al. [76] observed that the edge of the isolation membrane was often found next to ER exit sites, the sites of COPII vesicle formation. Interestingly, Atg9 and the Atg2-Atg18 complex accumulated at the edge of the isolation membrane. Consistent with this, Graef et al. identified protein-protein interactions between Atg and COPII coat proteins using a proteomics approach, and showed that the edge of the isolation membrane is located in close proximity to the ER exit sites [77]. Although the exact mechanism of how ER exit sites contribute to autophagosome biogenesis has yet to be elucidated, it has previously been shown that ER-toGolgi trafficking plays an important role in autophagosome biogenesis both in yeast $[78,79]$ and in mammals $[80,81]$.

\section{The ER-Golgi intermediate compartment (ERGIC)}

Recently, the ERGIC was proposed as another membrane source for autophagosome biogenesis. Ge et al. [82] found that the membrane fraction from Atg5-knockout MEF cells, in which no LC3 conjugation occurs, can be conjugated with LC3 when incubated with the cytosol from wild type cells. Using this cell-free assay system, they further performed membrane fractionation and found that LC3 was preferentially conjugated with the membrane subfraction containing ERGIC marker proteins. Consistent with this, drugs that disrupt ERGIC also suppressed LC3 conjugation and LC3 dot formation. Expression of Sar1A mutants that inhibit COPII vesicle formation also suppressed LC3 dot formation. In addition, ERGIC disruption resulted in reduced membrane recruitment of $\operatorname{Atg} 14 \mathrm{~L}$. In their assay system, the MAM fraction was not preferentially conjugated with LC3 even though starvation-induced Atg14L enrichment in this fraction [68] was confirmed, suggesting that the MAM fraction alone is not the primary target of LC3 conjugation. The preferential LC3 conjugation in the ERGIC fraction does not appear to be the consequence of the enrichment of PE or Atg proteins (ULK1, Atg7, Atg3, Atg16, Atg14, and Beclin 1 were mostly found in the cytosol, and Atg9 was not enriched in the ERGIC fraction). This indicates that other factors required for LC3 conjugation are enriched in the ERGIC fraction, but not in the other membrane fractions or in the cytosol.

\section{The plasma membrane and recycling endosomes}

The plasma membrane has been suggested as one of the membrane sources for autophagosome formation. The group led by David Rubinsztein has shown that Atg16L1 interacts with the heavy chain of clathrin and localizes to clathrin-coated structures. Inhibition of clathrinmediated endocytosis decreased the number of Atg16L1 dots and autophagosomes [83]. Further study showed that the SNARE protein VAMP7 and its partner SNAREs (syntaxin 7, syntaxin 8, and Vtilb) localize to Atg16L1positive vesicles, and mediate the homotypic fusion of these vesicles. The knockdown of these SNARE proteins inhibits autophagosome formation, suggesting that Atg16L1-positive vesicles may mature into the isolation membrane through homotypic fusion [84].

Recycling endosomes are also implicated in autophagosome formation. The group led by Sharon Tooze has shown that Atg9 partially colocalizes with recycling endosomes [40] in addition to the previously reported colocalization of Atg9 with the trans-Golgi network and other types of endosomes [26, 85]. They also identified TBC1D14 in a screen for putative RabGAP proteins involved in autophagy. They found that TBC1D14 binds to Rab11, a resident of recycling endosomes. Although TBC1D14 has a putative RabGAP domain, it does not appear to have RabGAP activity for Rab11, instead it may act as a downstream effector of Rab11. Overexpression of TBC1D14 negatively regulates autophagy possibly by sequestering the ULK1 complex in the recycling endosome through the direct binding between TBC1D14 and ULK1 [86].

In contrast to this, sorting nexin 18 (SNX18) has been identified as a positive regulator of autophagy in an RNAi screen for PX domain-containing proteins regulating autophagy [87]. SNX18 has a BAR domain, which can sense/induce membrane curvature, and overexpression of SNX18 causes formation of LC3- and Atg16L- 
positive membrane tubules. SNX18 interacts with Atg16L1 through its C-terminal PX-BAR domain and directly binds LC3 through its N-terminal LC3-binding motif. Its PX domain also binds PI(4,5)P2. Since both SNX18 and Atg16L1 colocalized with recycling endosome markers (transferrin receptor and Rab11), it was proposed that recycling endosomes may supply membrane lipids for autophagosome formation.

Thus, both Atg9 and Atg16L1 have been shown to localize to recycling endosomes. Recently, the Rubinsztein group also reported that Atg9 vesicles traffic to the plasma membrane [88]. They showed that Atg9 on the plasma membrane is internalized in a dynamin- and AP2-dependent manner, similarly to Atg16L1 endocytosis they had previously reported. However, Atg9 and Atg16L1 were not found together in the same clathrincoated structures. Atg9, but not Atg16L1, trafficked through EEA1-positive early endosomes. Atg9 and Atg16L1 eventually colocalized in Rab11-positive recycling endosomes in a VAMP3-dependent manner. Clarification of how the Atg16L1- and Atg9-positive recycling endosomes contribute to autophagosome biogenesis is still pending investigation.

\section{Atg9 vesicles as a membrane source}

In mammals, Atg9 was shown to partially colocalize with LC3 by fluorescence microscopy [26]. However, immuno-electron microscopy analysis failed to detect Atg9 on autophagosomes [40]. Mammalian Atg9 may not be stably incorporated into isolation membranes or autophagosomes even though it may transiently interact with these structures. In contrast, however, yeast Atg9 has been detected on isolation membranes and autophagosomes, suggesting the incorporation of Atg9 vesicles into these membranes [57]. Interestingly, typically only a few Atg9 vesicles are recruited during one round of autophagosome formation in yeast [57]. Since so few Atg9 vesicles (30-60 nm diameter) cannot provide the lipid amount of one autophagosome (300-900 $\mathrm{nm}$ diameter), it is likely that other membrane sources exist. Nevertheless, fusion of Atg9 vesicles may provide the membrane platform that matures into the isolation membrane. Immunoelectron microscopy analysis in another study detected Atg9 in clusters of small vesicles and tubules, and it was proposed that the isolation membrane may be initiated by the fusion of these clustered vesicles/tubules [89]. The Atg9 vesicle fusion mechanism may involve the yeast Rab protein Ypt1 and its GEF complex (the TRAPP III complex), which are required for autophagy and were identified in purified Atg9 vesicles [90, 91]. In addition, SNARE proteins required for autophagosome formation in yeast have also been implicated in Atg9 vesicle fusion [92].

The recent structural analysis of yeast Atg17 provided further insights into the fusion mechanism of Atg9 vesicles [93]. Atg17 forms a crescent shape with an approximately $10 \mathrm{~nm}$ radius curvature, and two Atg 17 molecules bind each other at their C-termini to form an S-shaped homodimer. Since the radius of the Atg17 crescent is similar to that of Atg9 vesicles, the authors suspected that the Atg17 homodimer acts as a scaffold for two Atg9 vesicles and facilitates their fusion. However, the Atg17Atg31-Atg29 complex did not bind to liposomes in this study. It should be noted that the vesicles used in these experiments are synthetic liposomes and did not contain Atg9. In light of the previously reported Atg1-dependent Atg9-Atg17 interaction [94], the authors proposed that Atg9 or other molecules on the Atg9 vesicles may facilitate tethering of Atg9 vesicles by Atg17. In addition, they found that yeast $A \operatorname{tg} 1$ has a membrane-binding domain at its C-terminus similar to its mammalian ortholog ULK1 [29]. They named this domain the Early Autophagy Targeting/tethering domain. The vesicle-binding ability of this domain may further facilitate Atg9 vesicle fusion. This is still mostly hypothetical however, and should be tested experimentally.

\section{Autophagosome formation in selective autophagy}

In selective autophagy, the substrate is recognized by the isolation membrane and subjected to degradation. Selective autophagy targets intracellular cargoes such as pathogens (xenophagy), mitochondria (mitophagy), and protein aggregates (aggrephagy). In mammals, ubiquitination of a substrate is not only the targeting mechanism for the ubiquitin-proteasome pathway, but is also a common feature of selective autophagy [7]. For example, Parkin, an E3 ubiquitin ligase associated with Parkinson's disease, is required for mitophagy that occurs after mitochondrial damage induced by the uncoupling reagent CCCP. Parkin is recruited to mitochondria after CCCP treatment and ubiquitinates mitochondrial outer membrane proteins [95]. Likewise, LRSAM1 and Parkin have been identified as the E3 ubiquitin ligases required for xenophagy $[96,97]$. Moreover, ubiquitin tagging is sufficient to induce autophagic degradation of mRFP and peroxisomes [98]. It has been previously proposed that adaptor proteins such as p62, NDP52, NBR1, and optineurin mediate the association between the ubiquitinated substrate and the isolation membrane because these proteins can simultaneously bind ubiquitin and LC3 [99-102]. Adaptor proteins explain how the isolation membrane can exert its specificity for ubiquitinated substrates, but 
this still does not answer the question of how isolation membrane formation initiates in response to substrate recognition.

We have recently shown that early Atg proteins (ULK1, Atg9, and Atg16L1) are all recruited directly to intracellular Salmonella [85]. DFCP1 has also been shown to colocalize with LC3-positive Salmonella [103]. In addition, it was shown that early Atg proteins, including ULK1, Atg9, Atg14L, and WIPI1, are recruited to damaged mitochondria after CCCP treatment [56]. Interestingly, in the case of selective autophagy against Salmonella, the ULK1 complex, Atg9, and the Atg16L1 complex are recruited independently of each other, since knockout of one component does not abolish the recruitment of the other two [85]. Similarly, LC3 recruitment to damaged mitochondria is also independent of the ULK1 complex and Atg9 [56]. In starvation-induced autophagy, on the other hand, deletion of the ULK1 complex or Atg9 abolishes Atg16L1 complex dot formation [21, 56]. Therefore, the Atg recruitment hierarchy in selective autophagy appears different from that of canonical starvationinduced autophagy. In particular, the Atg16L1 complex must possess the ability to target substrates in a ULK1 complex- and Atg9-independent manner. Our recent finding that Atg16L1's C-terminal WD domain directly binds ubiquitin provides evidence for this [48]. Moreover, Atg16L1 was shown to directly bind the ULK1 complex subunit, FIP200, in this and two other studies [46, 47]. The binding of Atg16L1 to ubiquitin and FIP200 acts redundantly to direct $A \operatorname{tg} 16 \mathrm{~L} 1$ to the ubiquitinated substrate. It was also found that a ubiquitination inhibitor (E1 inhibitor) abolished the substrate recruitment of Atg proteins (ULK1, Atg9, Atg14L, WIPI1, Atg5, and LC3). Importantly, the recruitment of Atg proteins appears to be triggered by the membrane rupture of endosomes and the following ubiquitination of endosomal membrane proteins. Therefore, selective autophagy of intracellular pathogens may be triggered simply by recognizing endosomal membrane rupture caused by the pathogen inside the endosome, rather than by recognizing each pathogen in a pathogen type-specific manner. This model can be further supported by the fact that damaged lysosomes are selectively ubiquitinated and degraded by autophagy $[104,105]$. Taken together, the ubiquitin-dependent recruitment of early Atg proteins to substrates provides a model for isolation membrane formation during selective autophagy.

\section{Concluding remarks}

Since the discovery of Atg proteins, which enabled us to directly observe and manipulate the autophagic machinery, significant advances have been made in our understanding of the origin of the autophagosome. As discussed above, multiple organelles, including the ER, mitochondria, the Golgi, recycling endosomes, the plasma membrane, and the ERGIC, have been suggested to contribute to autophagosomal membrane biogenesis. In mammals, the platform for autophagosome biogenesis likely exists on the ER (Figure 2). The interaction with mitochondria may generate such a platform on the ER, and other proposed organelles may supply membrane to the forming isolation membrane. However, details in these processes, including the exact functions of Atg proteins in autophagosome biogenesis, mechanistic differences between starvation-induced and selective autophagy, lipid trafficking routes for autophagosomal membrane supply, and lipid compositions of autophagosomal membrane, are all still unclear and therefore await further investigations.

\section{Acknowledgments}

We thank our lab member Marija Landekic for discussion and English proofreading. Work in the authors' lab is supported by the Ministry of Education, Culture, Sports, Science and Technology (MEXT) of Japan and by Japan Science and Technology Agency CREST.

\section{References}

1 Geng J, Baba M, Nair U, Klionsky DJ. Quantitative analysis of autophagy-related protein stoichiometry by fluorescence microscopy. J Cell Biol 2008; 182:129-140.

2 Mizushima N, Yamamoto A, Hatano M, et al. Dissection of autophagosome formation using Apg5-deficient mouse embryonic stem cells. J Cell Biol 2001; 152:657-668.

3 Fujita N, Hayashi-Nishino M, Fukumoto H, et al. An Atg4B mutant hampers the lipidation of LC3 paralogues and causes defects in autophagosome closure. Mol Biol Cell 2008; 19:4651-4659.

4 Singh R, Cuervo AM. Autophagy in the cellular energetic balance. Cell Metab 2011; 13:495-504.

5 Baba M, Osumi M, Scott SV, Klionsky DJ, Ohsumi Y. Two distinct pathways for targeting proteins from the cytoplasm to the vacuole/lysosome. J Cell Biol 1997; 139:1687-1695.

6 Mizushima N, Ohsumi Y, Yoshimori T. Autophagosome formation in mammalian cells. Cell Struct Funct 2002; 27:421-429.

7 Fujita N, Yoshimori T. Ubiquitination-mediated autophagy against invading bacteria. Curr Opin Cell Biol 2011; 23:492497.

8 Kuma A, Hatano M, Matsui M, et al. The role of autophagy during the early neonatal starvation period. Nature 2004; 432:1032-1036.

9 Komatsu M, Waguri S, Ueno T, et al. Impairment of starvation-induced and constitutive autophagy in Atg7deficient mice. J Cell Biol 2005; 169:425-434. 
10 Saitoh T, Fujita N, Jang MH, et al. Loss of the autophagy protein Atg16L1 enhances endotoxin-induced IL-1beta production. Nature 2008; 456:264-268.

11 Sou Y, Waguri S, Iwata J, et al. The Atg8 conjugation system is indispensable for proper development of autophagic isolation membranes in mice. Mol Biol Cell 2008; 19:47624775.

12 Saitoh T, Fujita N, Hayashi T, et al. Atg9a controls dsDNAdriven dynamic translocation of STING and the innate immune response. Proc Natl Acad Sci USA 2009; 106:2084220846.

13 Tsukamoto S, Kuma A, Murakami M, Kishi C, Yamamoto A, Mizushima N. Autophagy is essential for preimplantation development of mouse embryos. Science 2008; 321:117-120.

14 Mizushima N, Komatsu M. Autophagy: renovation of cells and tissues. Cell 2011; 147:728-741.

15 Yang Z, Klionsky DJ. Eaten alive: a history of macroautophagy. Nat Cell Biol 2010; 12:814-822.

16 Klionsky DJ, Cregg JM, Dunn WA Jr, et al. A unified nomenclature for yeast autophagy-related genes. Dev Cell 2003; 5:539-545.

17 Nakatogawa H, Suzuki K, Kamada Y, Ohsumi Y. Dynamics and diversity in autophagy mechanisms: lessons from yeast. Nat Rev Mol Cell Biol 2009; 10:458-467.

18 Suzuki K, Kirisako T, Kamada Y, Mizushima N, Noda T, Ohsumi Y. The pre-autophagosomal structure organized by concerted functions of APG genes is essential for autophagosome formation. EMBO J 2001; 20:5971-5981.

19 Suzuki K, Kubota Y, Sekito T, Ohsumi Y. Hierarchy of Atg proteins in pre-autophagosomal structure organization. Genes Cells 2007; 12:209-218.

20 Itakura E, Mizushima N. Characterization of autophagosome formation site by a hierarchical analysis of mammalian Atg proteins. Autophagy 2010; 6:764-776.

21 Hara T, Takamura A, Kishi C, et al. FIP200, a ULKinteracting protein, is required for autophagosome formation in mammalian cells. J Cell Biol 2008; 181:497-510.

22 Kabeya Y, Noda NN, Fujioka Y, Suzuki K, Inagaki F, Ohsumi Y. Characterization of the Atg17-Atg29-Atg31 complex specifically required for starvation-induced autophagy in Saccharomyces cerevisiae. Biochem Biophys Res Commun 2009; 389:612-615.

23 Kabeya Y, Kamada Y, Baba M, Takikawa H, Sasaki M, Ohsumi Y. Atg17 functions in cooperation with Atg1 and Atg13 in yeast autophagy. Mol Biol Cell 2005; 16:25442553.

24 Kamada Y, Funakoshi T, Shintani T, Nagano K, Ohsumi M, Ohsumi Y. Tor-mediated induction of autophagy via an Apg1 protein kinase complex. J Cell Biol 2000; 150:1507-1513.

25 Chan EYW, Kir S, Tooze SA. siRNA screening of the kinome identifies ULK1 as a multidomain modulator of autophagy. J Biol Chem 2007; 282:25464-25474.

26 Young ARJ, Chan EYW, Hu XW, et al. Starvation and ULK1-dependent cycling of mammalian Atg9 between the TGN and endosomes. J Cell Sci 2006; 119:3888-3900.

27 Kawamata T, Kamada Y, Kabeya Y, Sekito T, Ohsumi Y. Organization of the pre-autophagosomal structure responsible for autophagosome formation. Mol Biol Cell 2008; 19:20392050 .
28 Cheong H, Nair U, Geng J, Klionsky DJ. The Atg1 kinase complex is involved in the regulation of protein recruitment to initiate sequestering vesicle formation for nonspecific autophagy in Saccharomyces cerevisiae. Mol Biol Cell 2008; 19:668-681.

29 Chan EYW, Longatti A, McKnight NC, Tooze SA. Kinaseinactivated ULK proteins inhibit autophagy via their conserved C-terminal domains using an Atg13-independent mechanism. Mol Cell Biol 2009; 29:157-171.

30 Obara K, Noda T, Niimi K, Ohsumi Y. Transport of phosphatidylinositol 3-phosphate into the vacuole via autophagic membranes in Saccharomyces cerevisiae. Genes Cells 2008; 13:537-547.

31 Kihara A, Noda T, Ishihara N, Ohsumi Y. Two distinct Vps34 phosphatidylinositol 3-kinase complexes function in autophagy and carboxypeptidase Y sorting in Saccharomyces cerevisiae. J Cell Biol 2001; 152:519-530.

32 Liang XH, Jackson S, Seaman M, et al. Induction of autophagy and inhibition of tumorigenesis by beclin 1. Nature 1999; 402:672-676.

33 Kihara A, Kabeya Y, Ohsumi Y, Yoshimori T. Beclin-phosphatidylinositol 3-kinase complex functions at the transGolgi network. EMBO Rep 2001; 2:330-335.

34 Zhong Y, Wang QJ, Li X, et al. Distinct regulation of autophagic activity by Atg14L and Rubicon associated with Beclin 1-phosphatidylinositol-3-kinase complex. Nat Cell Biol 2009; 11:468-476.

35 Matsunaga K, Saitoh T, Tabata K, et al. Two Beclin 1-binding proteins, Atg14L and Rubicon, reciprocally regulate autophagy at different stages. Nat Cell Biol 2009; 11:385-396.

36 Sun Q, Fan W, Chen K, Ding X, Chen S, Zhong Q. Identification of Barkor as a mammalian autophagy-specific factor for Beclin 1 and class III phosphatidylinositol 3-kinase. Proc Natl Acad Sci USA 2008; 105:19211-19216.

37 Itakura E, Kishi C, Inoue K, Mizushima N. Beclin 1 forms two distinct phosphatidylinositol 3-kinase complexes with mammalian Atg14 and UVRAG. Mol Biol Cell 2008; 19:5360-5372.

38 Reggiori F, Tucker KA, Stromhaug PE, Klionsky DJ. The Atg1-Atg13 complex regulates Atg9 and Atg23 retrieval transport from the pre-autophagosomal structure. Dev Cell 2004; 6:79-90.

39 Lu Q, Yang P, Huang X, et al. The WD40 repeat PtdIns(3)Pbinding protein EPG-6 regulates progression of omegasomes to autophagosomes. Dev Cell 2011; 21:343-357.

40 Orsi A, Razi M, Dooley HC, et al. Dynamic and transient interactions of Atg9 with autophagosomes, but not membrane integration, are required for autophagy. Mol Biol Cell 2012; 23:1860-1873.

41 Mizushima N, Noda T, Yoshimori T, et al. A protein conjugation system essential for autophagy. Nature 1998; 395:395398.

42 Ishibashi K, Fujita N, Kanno E, et al. Atg16L2, a novel isoform of mammalian Atg16L that is not essential for canonical autophagy despite forming an Atg12-5-16L2 complex. Autophagy 2011; 7:1500-1513.

43 Fujita N, Saitoh T, Kageyama S, Akira S, Noda T, Yoshimori T. Differential involvement of Atg16L1 in Crohn disease and canonical autophagy: analysis of the organization of 
the Atg16L1 complex in fibroblasts. J Biol Chem 2009; 284:32602-32609.

44 Fujioka Y, Noda NN, Nakatogawa H, Ohsumi Y, Inagaki F. Dimeric coiled-coil structure of Saccharomyces cerevisiae Atg16 and its functional significance in autophagy. $J$ Biol Chem 2010; 285:1508-1515.

45 Fujita N, Itoh T, Omori H, Fukuda M, Noda T, Yoshimori T. The Atg16L complex specifies the site of LC3 lipidation for membrane biogenesis in autophagy. Mol Biol Cell 2008; 19:2092-2100.

46 Gammoh N, Florey O, Overholtzer M, Jiang X. Interaction between FIP200 and ATG16L1 distinguishes ULK1 complex-dependent and -independent autophagy. Nat Struct Mol Biol 2012; 20;144-149.

47 Nishimura T, Kaizuka T, Cadwell K, et al. FIP200 regulates targeting of Atg16L1 to the isolation membrane. EMBO Rep 2013; 14:284-291.

48 Fujita N, Morita E, Itoh T, et al. Recruitment of the autophagic machinery to endosomes during infection is mediated by ubiquitin. J Cell Biol 2013; 203:115-128.

49 Romanov J, Walczak M, Ibiricu I, et al. Mechanism and functions of membrane binding by the Atg5-Atg12/Atg16 complex during autophagosome formation. EMBO J 2012; 31:4304-4317.

50 Hanada T, Noda NN, Satomi Y, et al. The Atg12-Atg5 conjugate has a novel E3-like activity for protein lipidation in autophagy. J Biol Chem 2007; 282:37298-37302.

51 Kirisako T, Ichimura Y, Okada $\mathrm{H}$, et al. The reversible modification regulates the membrane-binding state of Apg8/ Aut7 essential for autophagy and the cytoplasm to vacuole targeting pathway. J Cell Biol 2000; 151:263-276.

52 Ichimura Y, Kirisako T, Takao T, et al. A ubiquitin-like system mediates protein lipidation. Nature 2000; 408:488492.

53 Kirisako T, Baba M, Ishihara N, et al. Formation process of autophagosome is traced with Apg8/Aut7p in yeast. $J$ Cell Biol 1999; 147:435-446.

54 Kabeya Y, Mizushima N, Ueno T, et al. LC3, a mammalian homologue of yeast Apg8p, is localized in autophagosome membranes after processing. EMBO J 2000; 19:5720-5728.

55 Nakatogawa H, Ichimura Y, Ohsumi Y. Atg8, a ubiquitinlike protein required for autophagosome formation, mediates membrane tethering and hemifusion. Cell 2007; 130:165178.

56 Itakura E, Kishi-Itakura C, Koyama-Honda I, Mizushima N. Structures containing Atg9A and the ULK1 complex independently target depolarized mitochondria at initial stages of Parkin-mediated mitophagy. J Cell Sci 2012; 125:1488-1499.

57 Yamamoto H, Kakuta S, Watanabe TM, et al. Atg9 vesicles are an important membrane source during early steps of autophagosome formation. J Cell Biol 2012; 198:219-233.

58 Geng J, Nair U, Yasumura-Yorimitsu K, Klionsky DJ. Post-Golgi Sec proteins are required for autophagy in Saccharomyces cerevisiae. Mol Biol Cell 2010; 21:22572269.

59 Van der Vaart A, Griffith J, Reggiori F. Exit from the Golgi is required for the expansion of the autophagosomal phagophore in yeast Saccharomyces cerevisiae. Mol Biol
Cell 2010; 21:2270-2284

60 Ohashi Y, Munro S. Membrane delivery to the yeast autophagosome from the Golgi-endosomal system. Mol Biol Cell 2010; 21:3998-4008.

61 Yen W-L, Shintani T, Nair U, et al. The conserved oligomeric Golgi complex is involved in double-membrane vesicle formation during autophagy. J Cell Biol 2010; 188:101-114.

62 Shirahama-Noda K, Kira S, Yoshimori T, Noda T. TRAPPIII is responsible for the vesicular transport from early endosomes to the Golgi apparatus that facilitates Atg9 cycling in autophagy. J Cell Sci 2013; 126(Pt 21):4963-4973.

63 Axe EL, Walker SA, Manifava M, et al. Autophagosome formation from membrane compartments enriched in phosphatidylinositol 3-phosphate and dynamically connected to the endoplasmic reticulum. J Cell Biol 2008; 182:685-701.

64 Hayashi-Nishino M, Fujita N, Noda T, Yamaguchi A, Yoshimori T, Yamamoto A. A subdomain of the endoplasmic reticulum forms a cradle for autophagosome formation. Nat Cell Biol 2009; 11:1433-1437.

65 Ylä-Anttila P, Vihinen H, Jokitalo E, Eskelinen E-L. 3D tomography reveals connections between the phagophore and endoplasmic reticulum. Autophagy 2009; 5:1180-1185.

66 Matsunaga K, Morita E, Saitoh T, et al. Autophagy requires endoplasmic reticulum targeting of the PI3-kinase complex via Atg14L. J Cell Biol 2010; 190:511-521.

67 Fan W, Nassiri A, Zhong Q. Autophagosome targeting and membrane curvature sensing by Barkor/Atg14(L). Proc Natl Acad Sci USA 2011; 108:7769-7774.

68 Hamasaki M, Furuta N, Matsuda A, et al. Autophagosomes form at ER-mitochondria contact sites. Nature 2013; 495:389393.

69 Koyama-Honda I, Itakura E, Fujiwara TK, Mizushima N. Temporal analysis of recruitment of mammalian ATG proteins to the autophagosome formation site. Autophagy 2013; 9:1491-1499.

70 Bodemann BO, Orvedahl A, Cheng T, et al. RalB and the exocyst mediate the cellular starvation response by direct activation of autophagosome assembly. Cell 2011; 144:253267.

71 Karanasios E, Stapleton E, Manifava M, et al. Dynamic association of the ULK1 complex with omegasomes during autophagy induction. J Cell Sci 2013 Sep 6. doi:10.1242/ jes. 132415

72 Tian Y, Li Z, Hu W, et al. C. elegans screen identifies autophagy genes specific to multicellular organisms. Cell 2010; 141:1042-1055.

73 Hailey DW, Rambold AS, Satpute-Krishnan P, et al. Mitochondria supply membranes for autophagosome biogenesis during starvation. Cell 2010; 141:656-667.

74 Rowland AA, Voeltz GK. Endoplasmic reticulum-mitochondria contacts: function of the junction. Nat Rev Mol Cell Biol 2012; 13:607-625.

75 Itakura E, Kishi-Itakura C, Mizushima N. The hairpin-type tail-anchored SNARE syntaxin 17 targets to autophagosomes for fusion with endosomes/lysosomes. Cell 2012; 151:12561269.

76 Suzuki K, Akioka M, Kondo-Kakuta C, Yamamoto H, Ohsumi Y. Fine mapping of autophagy-related proteins during autophagosome formation in Saccharomyces cerevisiae. $J$ 
Cell Sci 2013; 126:2534-2544.

77 Graef M, Friedman JR, Graham C, Babu M, Nunnari J. ER exit sites are physical and functional core autophagosome biogenesis components. Mol Biol Cell 2013; 24:2918-2931.

78 Hamasaki M, Noda T, Ohsumi Y. The early secretory pathway contributes to autophagy in yeast. Cell Struct Funct 2003; 28:49-54.

79 Ishihara N, Hamasaki M, Yokota S, et al. Autophagosome requires specific early Sec proteins for its formation and NSF/ SNARE for vacuolar fusion. Mol Biol Cell 2001; 12:36903702.

80 Guo Y, Chang C, Huang R, Liu B, Bao L, Liu W. AP1 is essential for generation of autophagosomes from the transGolgi network. J Cell Sci 2012; 125:1706-1715.

81 Zoppino FCM, Militello RD, Slavin I, Alvarez C, Colombo MI. Autophagosome formation depends on the small GTPase Rab1 and functional ER exit sites. Traffic 2010; 11:12461261.

82 Ge L, Melville D, Zhang M, Schekman R. The ER-Golgi intermediate compartment is a key membrane source for the LC3 lipidation step of autophagosome biogenesis. Elife 2013; 2:e0947.

83 Ravikumar B, Moreau K, Jahreiss L, Puri C, Rubinsztein DC. Plasma membrane contributes to the formation of preautophagosomal structures. Nat Cell Biol 2010; 12:747-757.

84 Moreau K, Ravikumar B, Renna M, Puri C, Rubinsztein DC. Autophagosome precursor maturation requires homotypic fusion. Cell 2011; 146:303-317.

85 Kageyama S, Omori H, Saitoh T, et al. The LC3 recruitment mechanism is separate from Atg9L1-dependent membrane formation in the autophagic response against Salmonella. Mol Biol Cell 2011; 22:2290-2300.

86 Longatti A, Lamb CA, Razi M, Yoshimura S-I, Barr FA, Tooze SA. TBC1D14 regulates autophagosome formation via Rab11- and ULK1-positive recycling endosomes. J Cell Biol 2012; 197:659-675.

87 Knævelsrud H, Søreng K, Raiborg C, et al. Membrane remodeling by the PX-BAR protein SNX18 promotes autophagosome formation. J Cell Biol 2013; 202:331-349.

88 Puri C, Renna M, Bento CF, Moreau K, Rubinsztein DC. Diverse autophagosome membrane sources coalesce in recycling endosomes. Cell 2013; 154:1285-1299.

89 Mari M, Griffith J, Rieter E, Krishnappa L, Klionsky DJ, Reggiori F. An Atg9-containing compartment that functions in the early steps of autophagosome biogenesis. J Cell Biol 2010; 190:1005-1022.

90 Lynch-Day MA, Bhandari D, Menon S, et al. Trs85 directs a Ypt1 GEF, TRAPPIII, to the phagophore to promote autophagy. Proc Natl Acad Sci USA 2010; 107:7811-7816.
91 Kakuta S, Yamamoto H, Negishi L, Kondo-Kakuta C, Hayashi N, Ohsumi Y. Atg9 vesicles recruit vesicle-tethering proteins Trs85 and Ypt1 to the autophagosome formation site. J Biol Chem 2012; 287:44261-44269.

92 Nair U, Jotwani A, Geng J, et al. SNARE proteins are required for macroautophagy. Cell 2011; 146:290-302.

93 Ragusa MJ, Stanley RE, Hurley JH. Architecture of the Atg17 complex as a scaffold for autophagosome biogenesis. Cell 2012; 151:1501-1512.

94 Sekito T, Kawamata T, Ichikawa R, Suzuki K, Ohsumi Y. Atg17 recruits Atg9 to organize the pre-autophagosomal structure. Genes Cells 2009; 14:525-538.

95 Youle RJ, Narendra DP. Mechanisms of mitophagy. Nat Rev Mol Cell Biol 2011; 12:9-14.

96 Huett A, Heath RJ, Begun J, et al. The LRR and RING domain protein LRSAM1 is an E3 ligase crucial for ubiquitindependent autophagy of intracellular Salmonella typhimurium. Cell Host Microbe 2012; 12:778-790.

97 Manzanillo PS, Ayres JS, Watson RO, et al. The ubiquitin ligase parkin mediates resistance to intracellular pathogens. Nature 2013; 501:512-516.

98 Kim PK, Hailey DW, Mullen RT, Lippincott-Schwartz J. Ubiquitin signals autophagic degradation of cytosolic proteins and peroxisomes. Proc Natl Acad Sci USA 2008; 105:20567-20574.

99 Kirkin V, Lamark T, Sou YS, et al. A role for NBR1 in autophagosomal degradation of ubiquitinated substrates. Mol Cell 2009; 33:505-516.

100 Pankiv S, Clausen TH, Lamark T, et al. p62/SQSTM1 binds directly to Atg8/LC3 to facilitate degradation of ubiquitinated protein aggregates by autophagy. J Biol Chem 2007; 282:24131-24145.

101 Wild P, Farhan H, McEwan DG, et al. Phosphorylation of the autophagy receptor optineurin restricts Salmonella growth. Science 2011; 333:228-233.

102 Thurston TLM, Ryzhakov G, Bloor S, von Muhlinen N, Randow F. The TBK1 adaptor and autophagy receptor NDP52 restricts the proliferation of ubiquitin-coated bacteria. Nat Immunol 2009; 10:1215-1221.

103 Huang J, Birmingham CL, Shahnazari S, et al. Antibacterial autophagy occurs at PI(3)P-enriched domains of the endoplasmic reticulum and requires Rab1 GTPase. Autophagy 2011; 7:17-26.

104 Maejima I, Takahashi A, Omori H, et al. Autophagy sequesters damaged lysosomes to control lysosomal biogenesis and kidney injury. EMBO J 2013; 32:2336-2347.

105 Hung YH, Chen LM, Yang JY, Yang WY. Spatiotemporally controlled induction of autophagy-mediated lysosome turnover. Nat Commun 2013; 4:2111. 